\title{
Membranous Labyrinth
}

National Cancer Institute

\section{Source}

National Cancer Institute. Membranous Labyrinth. NCI Thesaurus. Code C33090.

Part of the inner ear contained within the bony labyrinth that contains endolymph, and houses specialized sensory receptors involved in hearing and equilibrium. 